\title{
Procedural Knowledge Versus Conceptual Knowledge of Fraction : An Indonesian Junior High School Case Study
}

\author{
W Ramadianti ${ }^{1}$, N Priatna ${ }^{2}$, Kusnandi $^{3}$ \\ \{windaramadianti@upi.edu ${ }^{1}$ \} \\ Universitas Pendidikan Indonesia, Jl. Dr. Setia Budhi No. 229, Bandung 40154, Indonesia ${ }^{1,2,3}$, \\ Universitas Muhammadiyah Bengkulu, Jl. Bali,Kota Bengkulu38119, Indonesia ${ }^{1}$
}

\begin{abstract}
Fractions is one of the basic topics in mathematics. Knowledge about fraction relates conceptual knowledge and procedural knowledge. Therefore, the instructions about fractions have to consider them. To investigate ways of conceptual knowledge and procedural knowledge at mathematics instruction in junior high school, we observed mathematics instruction in two different classes which were taught by different teachers. This research was conducted at a junior high school in Bengkulu, Indonesia. The results showed that procedural knowledge was discussed more than conceptual knowledge. In addition, the study also found that almost procedural knowledge has not yet been connected with conceptual knowledge.
\end{abstract}

Keywords: Conceptual knowledge, Procedural knowledge, Fraction

\section{Introduction}

Some researchers revealed that conceptual and procedural knowledge are important components in understanding mathematics [1]. Its mean that a person is said to have an understanding of mathematics when he has conceptual and procedural knowledge. Therefore, in the learning process, teachers must facilitate students to access conceptual and procedural knowledge [1].

Conceptual knowledge is related to "knowing why" and procedural knowledge is related to "knowing how". The definition of conceptual and procedural knowledge which is often referred to is the opinion of Hiebert \& Lefevre in 1986) [2]. They define conceptual knowledge as "knowledge that is rich in relationships. It can be thought of as a connected web of knowledge, a network in which the linking relationships are as prominent as the discrete pieces of information. Relationships pervade the individual facts and propositions so that all pieces of information are linked to some networks "[2]. Meanwhile, procedural knowledge is defined in two types. First, it is closely related to the symbol of a system and the syntax for the configuration of acceptable symbols. Second, it consists of rules for solving mathematical problems [2]. Furthermore, Haapasalo and Kadijevich (2000) noted that (1) procedural knowledge usually requires unconscious automatic steps, whereas conceptual knowledge usually requires conscious thinking, (2) procedural knowledge might also involve some conscious thinking (for example when one combines two rules without knowing why they did it) [3]. 
Some research results on fractions and other mathematical topics provide different recommendations about the relationship of conceptual and procedural knowledge. Some researchers conclude that conceptual knowledge is a prerequisite knowledge for procedural knowledge [4],[5]. In the other hand, other researchers show that students who have little conceptual knowledge can solve problems about fractions procedurally [4]. Furthermore, some other researchers argue that conceptual and procedural knowledge stimulate one another simultaneously and continuously [4],[5].

These differences indicate a learning process that can be done differently. According to NCTM (2000), teaching conceptual knowledge starts with giving students problems that require flexibility of thinking and encouraging students to make connections with what they learned previously when solving problems, as well as enabling them to expand their prior knowledge and apply it in new situations [1]. Meanwhile, Skemp (1987) argues that teaching procedural knowledge is related to teaching about different terms, symbols, and skills without building a deep understanding [1]. The way teachers teach certainly influences the mathematical knowledge obtained by students. The importance of conceptual and procedural knowledge in learning mathematics, especially fraction, encouraged us to conduct an analysis of the fraction learning process in junior high school in terms of conceptual and procedural knowledge.

\section{Methods}

To investigate the teaching conceptual and procedural knowledge of fraction, we conducted case study research at a junior high school in Bengkulu Indonesia. We observed instruction process which conducted by two teachers. We observed them as long as instruction of fraction. Observation data are grouped based on conceptual and procedural knowledge.

\section{Result and discussion}

\subsection{Result}

Fraction has been studied in elementary school, so according to both participants, fraction in junior high school are only repetition. The result of observation show that two teachers teach in the same topic. They begin learning from defining fraction, comparing fraction, and fraction operations (addition, subtraction, multiplication and division).

$1^{\text {st }}$ participant teaches the definition of fractions by asking students about "what is fraction". A student gives the answer "fractions are numbers consisting of numerators and denominators". $1^{\text {st }}$ participant did not immediately conclude the fraction definition of the student's answer. He drew a circle on the whiteboard as an illustration of a birthday cake which would be shared for some people. Next, he divides the area of the circle into four equal parts, and then shades one part as an illustration of the given cake. $1^{\text {st }}$ participant asks students 'what part of the cake is given? Some students answer a quarter, and others answer one. $1^{\text {st }}$ participant leads students to notice that there are four parts, and one part is given. Then $1^{\text {st }}$ participant wrote the fraction $1 / 4$ as a symbol that stated one part of four parts. Furthermore, $1^{\text {st }}$ participant stated " 1 " as a numerator, and "4" as a denominator. Then, $1^{\text {st }}$ participant shaded one part another, then stated two parts of the four parts so students stated it as fraction $2 / 4.1^{\text {st }}$ participant also reminded about fraction forms such as percent and decimal. 
To teach about comparing fractions, $1^{\text {st }}$ participant asks students questions, "For example, I have two cakes. The first I divided for two children, while the second I divided for ten children. Which part is bigger? " Almost students said that which was shared for two children. $1^{\text {st }}$ participant states that this is true, logically if one cake is divided for two children then each child gets more cake than a cake divided for ten children. Then, $1^{\text {st }}$ participant asked again "which one is bigger, ten cakes divided for 12 children or three cakes for seven children?". For this question, students cannot immediately answer correctly. $1^{\text {st }}$ participant then stated that "to sort or compare fraction, the step that must be taken is to equalize the denominator, because what is seen as larger or smaller is a numerator with the terms the denominator is the same". Then $1^{\text {st }}$ participant gives some examples and questions to be solved by students like Figure 1.

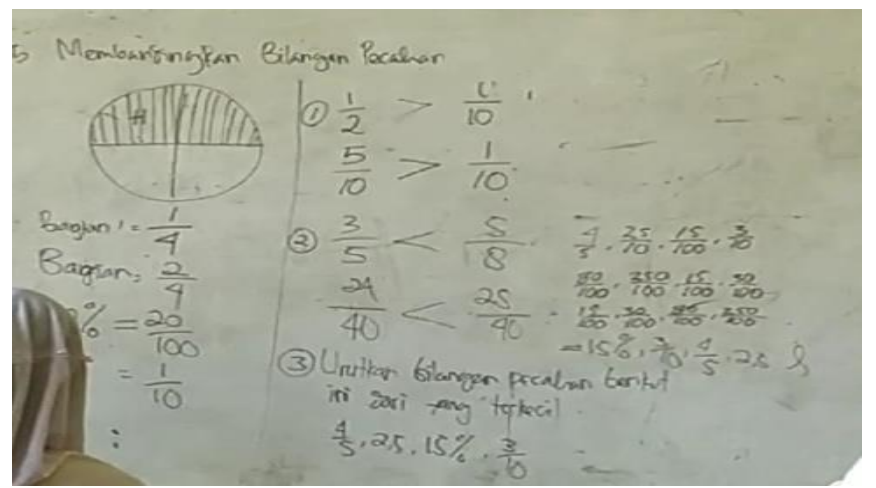

Fig. 1. Explanation about definition and comparing fraction of $1^{\text {st }}$ participant.

At the next meeting, $1^{\text {st }}$ participant taught the addition and subtraction operations on fractions. $1^{\text {st }}$ participant gives one example of addition and one example of subtraction. $1^{\text {st }}$ participant student recalled the process of adding and subtracting fractions. One of the students revealed how to equalize the denominator first, then it can be operated. According to $1^{\text {st }}$ participant, the opinion was incomplete, and asked other students to complete it. Another student stated that operated by numerator plus numerator. Finally, $1^{\text {st }}$ participant states "For addition and subtraction of fractions, if the denominator is same then the numerator and numerator can be added directly." For addition and subtraction with different denominators, participant one explains the use of the least common multiple of the denominator, and applies it to the fixed denominator. " Example problems given. Next, participant 1 gives examples of a wordy problem from a textbook to students to illustrate the application of addition and subtraction in daily life.

Next, $1^{\text {st }}$ participant taught about multiplication and division of fractiom. $1^{\text {st }}$ participant asks students to read the examples in the textbook in a few moments. Then, he and the students discussed the case in the example, that is, a pharmacist poured half a liter of liquid medicine every hour for five hours. They agreed that the case was an example of multiplication of fractions. Then the teacher asks students "how to multiply fractions". A student states how to multiply the numerator while the denominator remains. Other students refute the opinion. According to him the right way to multiply the denominator by the denominator and the numerator by the numerator. The teacher also states that the method is right. Then the class resolves the previous case by applying the agreed rules.

When teach about division operation, $1^{\text {st }}$ participant gives questions to students. Then, students are asked to explain how to solve the problem. One student expresses the way by replacing the for $(\div)$ sign with the times sign $(\times)$ and the fraction that is after the quotient is 
reversed. $1^{\text {st }}$ participant immediately confirmed the answer and applied the method in the example problem given earlier. Then, the teacher asks students to read one of the questions in a textbook, then he asks what operations are appropriate for solving the question. Together, students answer the division operation. Then he invited a student to write their solution on the board.

Meanwhile, $2^{\text {nd }}$ participant starts learning about fraction by asking students to read examples of cases related to comparing the following fractions. "In a birthday event, invitations that came were divided into 4 groups to enjoy a circle of cake of the same size. The tarts have been served at each group table, namely table A, table B, table C, and table D. The cake is shared equally with children who face a table. Every invitation that comes may choose to sit on any desk bench. Adit was the last invitation to come at the event. Adit saw that table A had had 6 children, table $\mathrm{B}$ there were 7 children, table $\mathrm{C}$ there were 8 children, and table D there were 9 children. (a) If Adit chooses to join the bench on table B, will many pieces of cake that Adit will get will be the same as the child who chooses which table? Explain (b) If Adit wants to get the most cake from among the four chosen tables, which table should Adit choose? Explain. "

Then they discussed the case. Based on the results of the discussion obtained fractions $1 / 8$ related to the first problem and $1 / 7$ related to the second problem. The teacher then asks "which is bigger $1 / 7$ or $1 / 8$ ?" Students answer $1 / 7$ greater. One student gave a reason because 7 people were fewer than 8 people, so that the cake that was obtained was more. The teacher justifies the opinion of the student. Next, the teacher asks students to look at the fractional illustration, and the teacher explains the purpose of the illustration.

Then, the teacher asks students to look at an example in a textbook about comparing two fraction. In this example, students are asked to determine "which is greater than, $2 / 3$ or $3 / 4$ ?".. Some students answer the same, but others answer different. The teacher asks students to look for information in a book related to how to compare it. A student reads the information and he found in a textbook about comparing $2 / 3$ and $3 / 4$. The teacher recounts the purpose of the information. The first step determines the least common multiple of 3 and 4 , which is 12 , after the denominator is changed to 12 , then the numerator is changed to 8 and 9 , so that the fractions are $8 / 12$ and $9 / 12$, and finally it can be determined that $2 / 3$ is more smaller than $3 / 4$. Next, the teacher concludes the steps of comparing fractions, which is looking for the least common multiple of the denominator, after the denominator is the same, compare the numerator. Then the teacher gives some similar questions as an exercise.

At the beginning of learning about the addition and subtraction, $2^{\text {nd }}$ participant gives 4 examples regarding addition and subtraction. The teacher conveys that for addition and subtraction of fractions with the same denominator can be completed by directly operating the numerator, whereas for addition and subtraction of fractions by different denominators, the least common multiple of the denominator must be determined to equalize the denominator. Next, the teacher asks students to do some exercises.

In learning about multiplication and division of fractions, the teacher asks one of the students to read the description in the textbook. Then, they discuss the same example with the first participant class. Then, they resolved the case. $2^{\text {nd }}$ participant presents the example which is an example of the operation of multiplication of fractions and integers. Next, he gives the example of multiplication which involves two fractions. To explain this, $2^{\text {nd }}$ participant asked students to pay attention to the illustration of the multiplication of fractions in the textbook. The teacher states that the part affected by shading twice shows the results of multiplying these two fractions. The teacher then links the illustration to the step of multiplying fractions by multiplying the numerator by the numerator and the denominator by the denominator. 
Then, $2^{\text {nd }}$ participant taught about the division of fractions. He immediately gave examples of operations $1 / 3: 2$. Two students write their opinions on the board, then the teacher discusses and writes the right answers (see Figure 2). Next, the teacher asks students to do some practice exercises.

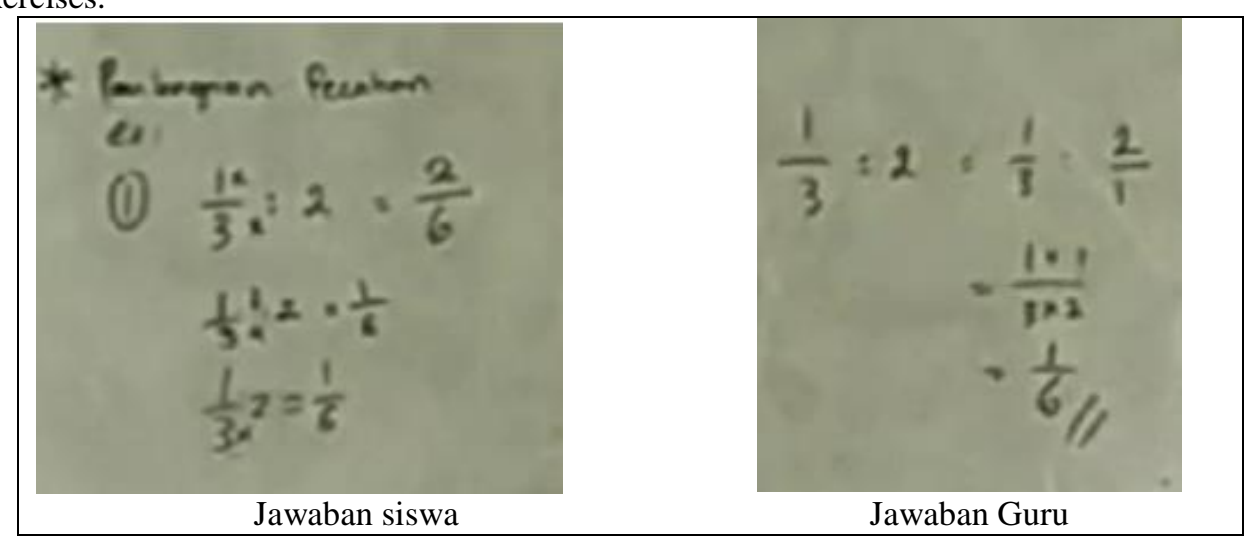

Fig. 2.Illustration of the multiplication of a fraction by $2^{\text {nd }}$ participant.

\section{2 Discussion}

Overall instruction by $1^{\text {st }}$ participant and $2^{\text {nd }}$ participant are no different. When teaching the definition of fractions, they describe fractions as part of the whole. The illustrations used are also the same, namely shading a number of parts which are then expressed as fractions. In this way, the teacher teaches procedural knowledge to students, because without them knowing it, they provide a special rule for writing a fraction, namely the denominator to state the number of all parts, and the numerator to state the many parts that are shaded. Yet the meaning of the symbol $a b$ as a fraction cannot only be interpreted as part of the whole. Besides part of the whole, fractions can also be interpreted as quotient, as ratio, as operators, and as measures. [6, $7,8]$. This incomplete definition of fraction results in the loss of meaning of fraction operations.

In learning about comparing fractions and fraction operations, both teachers also tend to emphasize the step of completing fraction operations. In learning to compare two fractions the rule used is to change both fractions so that they have the same denominator and then compare the numerator. This method becomes procedural knowledge for students. Actually conceptual knowledge related to comparing two fractions has been conveyed by the teacher by giving an illustration of a cake that is distributed to several people. Unfortunately, this is only limited to the case of fractions with the numerator "1". Whereas the use of the definition of part of the whole is not only limited to fractions with numerator 1. In addition, the teacher unconsciously, the illustrations they provide change the meaning of fractions from as part of the whole to as quotient, because of the teacher's explanation that is considered is that many cakes are received each each person.

In learning about the addition and subtraction operations of fractions, the two teachers immediately provide a procedure for adding or subtracting, namely by first equating the denominator without them knowing why the brand should equate the denominator. According to Behr, et. al (1983), the meaning of fractions in addition operations is as a measure [7],[9]. The illustration that can be used is the sum of the sizes on the number lines. With this illustration, the process of equating the denominator can be interpreted as the process of partitioning a number line. 
Likewise in multiplication and division operations, learning is more focused on procedural knowledge, so fraction multiplication operations are also meaningless. According to Behr, et. al (1983) the meaning of fractions in multiplication operations is as an operator [7],[9]. In the multiplication of two fractions, one of them is the operator, which is the first fraction. For example, we often translate multiplication operations of $1 / 3 \times 2 / 3$ to $1 / 3$ of $2 / 3$. This actually shows that $1 / 3$ is an operator that processes $2 / 3$. This results in a different role between the first fraction and the second fraction in multiplication operations, although the commutative nature still applies to the product of the two fractions. Actually, $2^{\text {nd }}$ teacher has illustrated this by paying attention to Figure 3, but the limitations of the definition of fractions at the beginning of learning have an impact on the meaning of fraction multiplication. Furthermore, the division of fractions can be seen as the opposite of the fraction multiplication.

Based on the results of observations, mathematics learning conducted at participants' schools has provided students with appropriate procedural knowledge. This can be knowledge that students can use to solve procedural problems. However, it does not seem that the relationship between materials indicates that students still do not have good conceptual knowledge about fractions.

\section{Conclusion}

The following conclusions from this study. Learning about fraction in junior high school consists of the definition of fractions, comparing fractions, and fraction operations. The definition of fraction learned is fraction as part of whole. Learning about comparing fractions and fraction operations is focused on procedural knowledge, and does not yet involve conceptual knowledge.

\section{References}

[1] Khashan, K. H.: Conceptual and Procedural Knowledge of Rational Numbers for Riyadh Elementary. Journal of Education and Human Development . Vol 3 (4), pp. 181 - 197.(2014)

[2] Star, J. R., and Stylianides, G. J.: Procedural and Conceptual Knowledge: Exploring the Gap Between Knowledge Type and Knowledge Quality. Canadian Journal of Science, Mathematics, and Technology Education. Vol 13 (2), pp. 169-181. (2013)

[3] Kadijevich, D. M.: Relating Procedural and Conceptual Knowledge. The Teaching of Mathematics. Vol 21 (1), pp. 15 -28. (2018)

[4] Hallet, D , and Nunes T.: Individual differences in conseptual and procedural knowledge when learning fraction. Journal of Educational Psychology. Vol 102 (2), pp. 395 - 406. (2010)

[5] Schneide, M. and Stern, E.: Conceptual and Procedural Knowledge of Mathematics Problem: Their Measurement and Their Causal Interrelation. (2005)

[6] Chapin SH and Johnson A.: Interpretation of fractions. Math Matter, 99 - 101. (2012)

[7] Pienaar E.: Learning about and understanding fractions and their role in the high school curriculum. Stellenbosch University. (2014)

[8] Lamon S.: Teaching fractions and ratios for understanding. (New York : Taylor \& Francis). (2012)

[9] Charalambous CY and Pitta-Pantazi D.: Drawing on a theoretical model to study students' understanding of fractions. Educational Studies in Mathematics, 64: 293-316. (2007) 